\title{
Paisaje e imaginarios urbanos: dinámicas en la construcción de identidad y sentido de lugar en Sincelejo, Colombia
}

\author{
Landscape and urban imaginaries: dynamics in the \\ construction of identity and sense of place in Sincelejo, \\ Colombia
}

\section{Resumen}

Autores:

Pedro A. Martínez-Osorio* pedro.martinez@cecar.edu.co Francisco H. Martínez-Ochoa** franciscomartinez@unisinu.edu.co

Alexandra Castellanos-Tuirán* alexandra.castellanos@cecar.edu.co

*Corporación Universitaria del Caribe **Universidad del Sinú

Colombia e analizan relaciones entre paisaje e imaginarios urbanos en una ciudad del Caribe colombiano, Sincelejo, a partir de la integración de resultados de $\checkmark$ dos investigaciones sobre el mismo objeto de estudio: el centro histórico. Una trabaja las representaciones sobre elementos del espacio público desde un enfoque fenomenológico; la otra, desde una aproximación cuantitativa con el objetivo de analizar las condiciones ambientales y paisajísticas del centro histórico, se enfoca en el inventario del arbolado urbano y el análisis de los servicios ambientales que ofrece a la ciudad. Entre los hallazgos destaca la relación de interdependencia entre paisaje y habitante, la formación y consolidación de identidades territoriales con relación al paisaje. El paisaje funciona como un catalizador para la satisfacción de los deseos de múltiples individuos. En este proceso, seres humanos y paisaje se funden aportando sentido al lugar, lo que convierte a esas dinámicas en un patrimonio a preservar y valorar en la ciudad.

Palabras clave: imaginarios, arquitectura del paisaje, patrimonio cultural, centro histórico, espacio público.

\section{Abstract:}

Relations between urban imaginary and landscape are analyzed in a Colombian Caribbean city, Sincelejo, based on the integration of the results of two investigations on the same object of study: the historic center. One works the representations on elements of the public space from a phenomenological approach; the other, from a quantitative approach with the objective of analyzing the environmental and landscape conditions of the historic center, focuses on the inventory of urban trees and the analysis of environmental services it offers to the city. Among the findings, the interdependence relationship between landscape and inhabitant stands out, as well as the formation and consolidation of territorial identities in relation to the landscape. The landscape works as a catalyst for the satisfaction of the desires of multiple individuals. In this process human beings and landscape merge, giving meaning to the place, which turns these dynamics into a heritage to preserve and value in the city.

Keywords: imaginary, landscape architecture, cultural heritage, city center, public space. 


\section{Introducción}

Muchos autores hablan sobre la complejidad del término paisaje debido a la gran cantidad de disciplinas que convoca. En la actualidad este término cobra gran importancia debido al interés creciente de realizar estudios urbanos con enfoque holístico, sensible e integrador, como instrumento para aportar información que permita conocer de una manera más profunda el territorio, y así desarrollar políticas públicas de ordenamiento en zonas específicas de la ciudad, más cercanas a las vivencias y realidades del entorno urbano.

El estudio de la ciudad a partir de la mirada integradora de diferentes disciplinas, arroja una información valiosa en la cual el paisaje hace parte indisoluble de las representaciones colectivas de un lugar. Se entiende el paisaje como un hecho complejo fundamentado en las interrelaciones de carácter social y cultural sobre una base natural, material y física de las estructuras urbanas (Nogué, 2010), así como una base espacial de carácter inmaterial.

También Maderuelo (2005) asimila el concepto de paisaje como una elaboración mental que los seres humanos realizan a través de los fenómenos de la cultura, un constructo que varía de una cultura a otra. En ese mismo sentido, es interesante el trabajo desarrollado por Pérez-Igualada y VicenteAlmazán (2020), ya que examinan la relación entre espacio, tiempo y paisaje desde dos perspectivas que son complementarias: la percepción y el proyecto

Por otra parte, Benedict y McMahon, (2012), a partir del concepto de infraestructura verde, en el que incluyen bosques, humedales, pantanos, manglares, praderas, zonas verdes, entre otros, destacan la importancia de estos elementos para el buen funcionamiento de la ciudad, por los servicios críticos que proveen diariamente en materia de integridad ecológica, esparcimiento, agua limpia, aire puro y alimentos. Para los autores las áreas verdes proveen servicios esenciales para la vida de todas las especies.

El estudio de Salazar (2010) acerca del uso y apropiación del espacio público, se cuestiona sobre la ocupación, usos, permanencias, posturas en el tiempo, y la apropiación del espacio público, considerando una serie de variables de carácter ambiental y bioclimático, así como otras variables de tipo sensible que se toman como referencia de formas de trabajo de otras disciplinas, como la antropología, geografía e historia. Es una interesante referencia, por la integración de diferentes saberes en la comprensión de las dinámicas que acontecen en el espacio público.
Uno de los conceptos claves para este trabajo es retomado de García-Canclini (1997), cuando se refiere a los imaginarios como elaboraciones simbólicas de lo que se observa; también los define como prácticas sociales desde las cuales los individuos de la ciudad reconstruyen el mundo y su estructura lógica, con el fin de aprehenderlo. Para García-Canclini, la ciudad es algo más que un fenómeno físico.

En la literatura existente sobre el tema de estudio, existe mucha información, sobre todo en el ámbito latinoamericano, donde se ha avanzado en el perfeccionamiento, cada vez más preciso, de metodologías cualitativas para el desarrollo de esta clase de estudios. Se puede destacar, entre el amplio espectro, el trabajo de Gorelik (2002), quien aporta la visión de la metáfora cartográfica para entender la ciudad. El trabajo de Silva (2006), quien propone la elaboración de una nueva cartografía para reconstruir el croquis de la ciudad, y de esa forma materializar la existencia de múltiples territorios (Silva, 2006, p. 66). También los trabajos de Gallardo (2012; 2013), que enfatizan sobre la importancia en la arquitectura de las reflexiones sobre el lugar y el habitar.

En la misma línea, Lindón (2007) aborda la posibilidad de realizar miradas alternativas en distintos tiempos y lenguajes sobre los imaginarios de la ciudad. Recoge ideas de diversos autores y traza un camino posible en la construcción de una metodología cada vez más diversa para estudios de este tipo, en la cual los imaginarios emergen tanto en los discursos como en otras expresiones del lenguaje social como obras de arte, cartas, grafitis, fotografías, etc. (Lindón, 2007).

Otros estudios que se consideran de gran importancia en el estado del arte de la investigación alrededor de este tema, son los desarrollados por De Certeau (1996), sobre las relaciones entre lugar y memoria. El autor sugiere diferencias entre los conceptos de espacio y lugar, en tanto que este último es depositario, y al mismo tiempo productor, de memorias y narrativas que nacen de las interacciones de los habitantes. También los estudios realizados por Martín-Barbero (2002), quien trabaja sobre las tensiones y discontinuidades presentes en las narrativas que hacen parte de las formas de aprehensión de la ciudad.

Son muy importantes las investigaciones de Juan Carlos Pérgolis, en las cuáles sus hipótesis de trabajo sugieren que la imagen urbana no pertenece a la ciudad, sino a sus habitantes, siendo este el modo como los ciudadanos la representan en su mente (Pérgolis y Valenzuela, 2008; Pérgolis y Rodríguez, 2014; Pérgolis y Quijano, 2019; Pérgolis, 2020).

Rojo y Enríquez (2010) definen los imaginarios urbanos como un esquema de significados dinámicos construidos socialmente. Para ellos la presencia de los imaginarios urbanos dan cuenta de acciones institucionalizadas que se inscriben en la ciudad, las cuales trabajan en una doble vía, en constante dinamismo entre lo material, los significados y quien los percibe.

Por otra parte, los estudios urbanos, sobre todo en las últimas décadas, al ser desarrollados desde enfoques 
cualitativos, han despertado el interés por su aporte a las municipalidades como instrumento para el desarrollo de sus políticas, esto debido a que develan interrogantes que hablan sobre la identidad y las dinámicas que se suceden en el ámbito urbano.

Cidell (2017) analiza las diferentes relaciones de los imaginarios y los conceptos de sostenibilidad en Chicago, lo que permite entender dinámicas urbanas útiles en la toma de decisiones. En el mismo sentido, trabajos como los de Borie, Pelling, Ziervogel y Hyams (2019), Gross, Buchanan y Sané (2019), se valen de las narrativas e imaginarios para empoderar y estimular la resiliencia de las comunidades.

La ciudad de Sincelejo, capital del departamento de Sucre, Colombia, es visionada en el mediano y largo plazo como una ciudad competitiva, con grandes inversiones del sector público y privado en el contexto regional, y con una reciente situación coyuntural para determinar el futuro de la ciudad, en la que se entrega la revisión al plan de ordenamiento territorial para el municipio (P.O.T). Esta situación es una oportunidad para trazar estrategias tendientes a la protección y valoración del patrimonio municipal, incluido en él las estructuras urbanas que hacen parte del centro histórico.

De acuerdo con el gran interés alrededor del centro de Sincelejo, su preservación y valoración, y teniendo en cuenta el gran vacío de conocimiento que existe sobre esas estructuras, se han desarrollado estudios enfocados en la comprensión del centro histórico de Sincelejo desde la participación de múltiples visiones, con el fin de preservar a futuro un lugar que hace parte de la identidad de la ciudad.

Este trabajo se desprende de la integración de los resultados de 2 investigaciones sobre ese objeto de estudio. Una toca el tema de las representaciones existentes sobre los elementos del espacio público que definen el centro de Sincelejo, desde un enfoque fenomenológico, construyendo relaciones y extrayendo el sentido del lugar de las dinámicas que actualmente se desenvuelven en ese entorno urbano. La otra plantea un análisis de las condiciones ambientales y paisajísticas del centro histórico, con un enfoque cuantitativo, centrado en el inventario del arbolado urbano y el análisis en cuanto a los servicios ambientales que ofrece este conjunto a la ciudad.

En este artículo se optó por presentar parte de los resultados de ambas investigaciones, debido a los aspectos interesantes en los cuales confluyen los dos estudios, además por entender que el patrimonio de un lugar está constituido por diversos elementos, entre los cuales se encuentran el agua, el suelo, la vegetación, las edificaciones, las costumbres, la memoria, etc., pero principalmente se configura a través de la interacción entre esos elementos, sobre todo en cuanto a la influencia en doble vía entre el paisaje y la sociedad, aspecto sobre el cual toda la sociedad es convocada a participar en su valoración, protección y salvaguarda (UNESCO, 1972; 1977; 2003; 2005).

Siguiendo esa línea de pensamiento, Aponte (2008) explica que: "El paisaje es la expresión perceptible de la interacción constante entre los lugares y la gente" (2008, p.4), sobre todo con la finalidad de analizar a través de la información obtenida la relación entre el paisaje y las representaciones de la sociedad con respecto a uno de sus lugares más identitarios.

\section{Métodos}

Se integran resultados de dos investigaciones, una cualitativa y otra cuantitativa. El trabajo cualitativo se desarrolló con una metodología de enfoque fenomenológico y hermenéutico, con el objetivo de analizar representaciones de la comunidad al respecto de uno de sus espacios públicos más distintivos. Se llevó a cabo en 4 fases: las fases 1 y 2, de tipo descriptivo, consistieron en el inventario de los elementos del espacio público y la identificación de percepciones e imaginarios urbanos. Se utilizaron para la recolección de información 3 tipos de fuentes primarias, dos encuestas de tipo abierto que trabajaban la identificación del área de estudio y las percepciones sobre los espacios públicos que definen el centro histórico de Sincelejo; una serie de representaciones gráficas del espacio público, realizadas por diversos actores de la comunidad, y una entrevista en profundidad realizada a personajes claves que se ubican en el espacio público del centro histórico de Sincelejo (figura 1).

La fase 3, de tipo analítico, se desarrolló con la intención de examinar los discursos de la población alrededor de los elementos del espacio público que definen el centro histórico. Se caracterizaron los tipos de discursos encontrados en las narraciones de los personajes. Se utilizó el software ATLAS TI 6.0, como herramienta de análisis de datos cualitativos. Se realizó una codificación primaria, que permitió establecer unas categorías axiales a partir de la codificación de textos, imágenes y relatos obtenidos en la recolección de información. Estas ayudaron a entender los significados en el nivel simbólico de las percepciones y experiencias de los habitantes en el espacio. A partir de las categorías, sus relaciones e interconexiones, se organizaron en forma de diagramas las percepciones de la comunidad al respecto de los elementos del espacio público que definen el centro de Sincelejo (Jacobs, 2013).

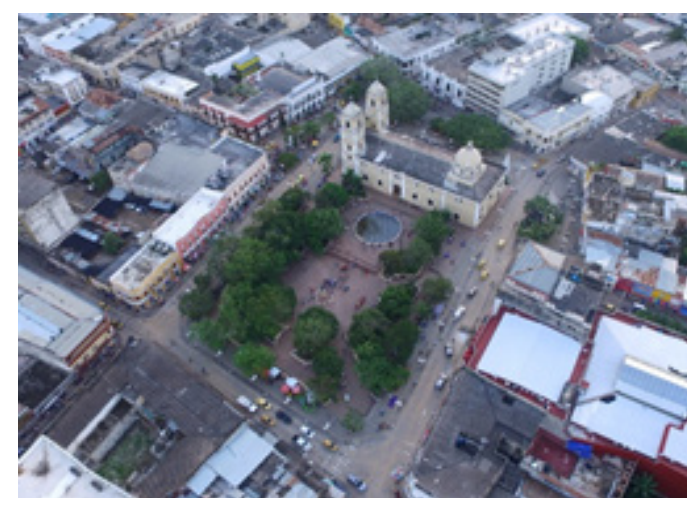

Figura 1: Parque Santander, centro histórico de Sincelejo. Fuente: Los autores (2016) 
La fase 4, de tipo comparativa, cuya intención fue contrastar las categorías de análisis que hacen parte de los discursos sobre el lugar de estudio con la realidad físico-espacial existente del lugar, permitió reconstruir una mapificación-croquis de las actividades existentes determinando recorridos, permanencias y espacializando las actividades categorizadas con respecto a la realidad del lugar (Gorelik, 2002).

La otra investigación, de tipo descriptivo y de enfoque cuantitativo, cuyo objetivo fue el análisis de las condiciones ambientales y paisajísticas del centro histórico, se centra en el inventario del arbolado urbano y el análisis en cuanto a los servicios ambientales que ofrece el conjunto ambiental y paisajístico a la ciudad. Toma como población todas las especies de árboles que hacen parte de la comuna cinco (ver figura 2); vincula también los transeptos que pasan por el centro histórico, del que hacen parte los arbolados localizados en los patios y terrazas de las casas, locales comerciales y demás inmuebles que están ubicados en dicho centro histórico. Fue desarrollada en tres fases: 1) Recolección y levantamiento de la información, 2) Sistematización y presentación del inventario, 3) Análisis ecológico y biológico del inventario.

El proceso de recolección de la información inició con el análisis de la ocupación y organización del territorio. Se identificaron los índices de ocupación y usos del suelo (revisión de cartas catastrales, zonificación a partir de imágenes aéreas, levantamiento de parques y zonas de esparcimiento al aire libre). Se realizó por medio de la medición y seguimiento de tres variables climáticas, temperatura, humedad relativa y luminosidad, durante los primeros seis meses del año 2015, consiguiendo los datos día a día en dos circunstancias diferentes: bajo la sombra arrojada por la copa de los árboles y fuera de la sombra de la copa de los árboles, en tres horas distintas del día: 7:00 am, 12:00 m y 5:00 pm (figuras 3 y 4). Todo con el objetivo de obtener un promedio de estas, para entender y comprender el concepto de microclima en un espacio abierto urbano, que en este caso viene a ser el parque Santander en el centro histórico de Sincelejo.

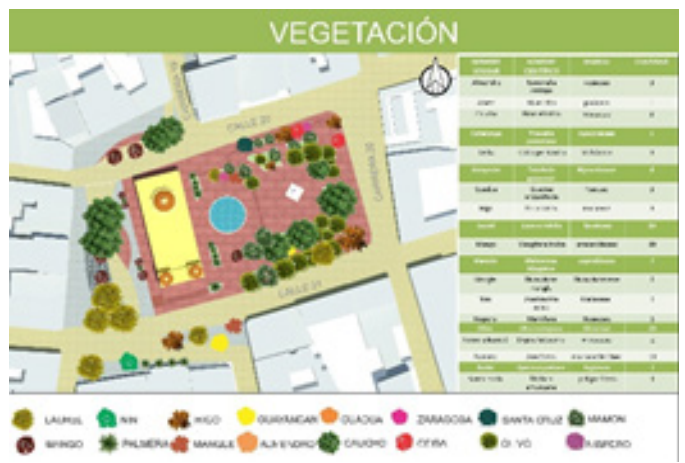

Figura 2: Inventario de la vegetación en la zona de estudio.

Fuente: Los autores (2016)

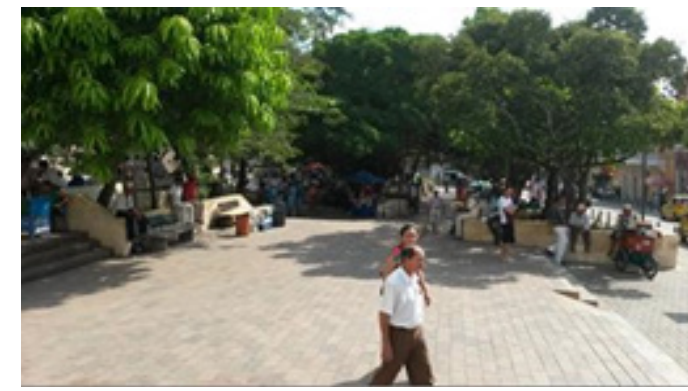

Figura 3: Vista general del lugar de estudio Fuente: Los autores (2015)

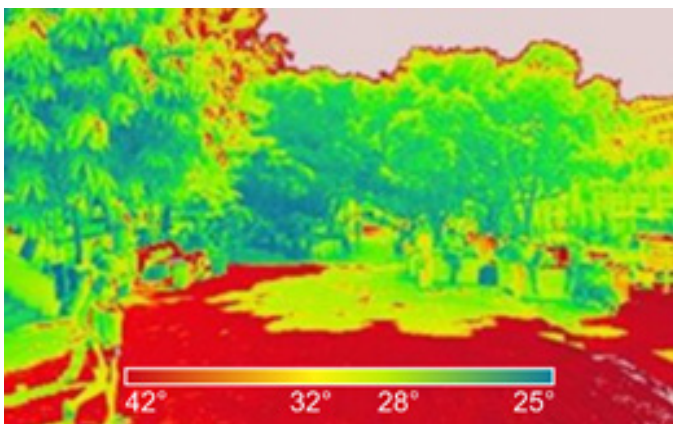

Figura 4: Vista termográfica del lugar de estudio Fuente: Los autores (2015)

\section{Resultados}

Con relación al proceso de acercamiento a las percepciones e imaginarios urbanos existentes sobre los elementos del espacio público que definen el centro histórico de Sincelejo, se identificaron y clasificaron las percepciones de la población al respecto de tales estructuras urbanas, con el fin de reconstruir significados a nivel simbólico, característicos de las representaciones sociales e imaginarios construidos por la comunidad.

La primera clasificación de las percepciones sobre los elementos del espacio público permitió identificar tres categorías axiales, a saber: categoría 1: "percepciones positivas", como aquellas percepciones de los consultados en las que se observa una idea positiva sobre el lugar y las estructuras urbanas que definen ese lugar. La categoría 2: "percepciones negativas", agrupa percepciones de los habitantes consultados desde las cuales se evidencia una idea negativa sobre los elementos y actividades que se dan en los espacios públicos que definen el centro histórico. Finalmente, la categoría 3: "sentimientos sobre el lugar", agrupa aquellas evaluaciones que los miembros de la población hacen al respecto de las percepciones y emociones que han experimentado en la vivencia del lugar.

Fue interesante, en el proceso, revisar las subcategorías que soportan las "percepciones positivas", las "percepciones negativas" y los "sentimientos sobre el 
lugar". En primera instancia aquellas que dan soporte a la percepción positiva sobre el lugar son: lo ambiental, lo funcional, la movilidad, el estado actual, la arquitectura y lo social. En otro sentido, las que dan soporte a la percepción negativa sobre el lugar son: la reducción del espacio público, obras inconclusas, pérdida de identidad, la gobernabilidad, lo social, la movilidad, la inseguridad y lo ambiental. Con relación a la categoría axial, denominada "sentimientos sobre el lugar", las tres subcategorías que la soportan son: armonía, melancolía e intranquilidad.

Es interesante observar que la subcategoría denominada "lo ambiental" aparece referenciada como soporte en ambos tipos de percepciones, tanto positivas como negativas, lo cual genera unas tensiones importantes en cuanto al ambiente como elemento que hace parte de las diferentes percepciones que se tienen sobre el lugar objeto de estudio.

Por una parte, en lo positivo, el ambiente se relaciona con percepciones conexas a la comodidad del lugar, la buena vegetación, la preservación de las mismas, el aire y la tranquilidad que el conjunto natural en el espacio urbano ofrece (ver figura 5).

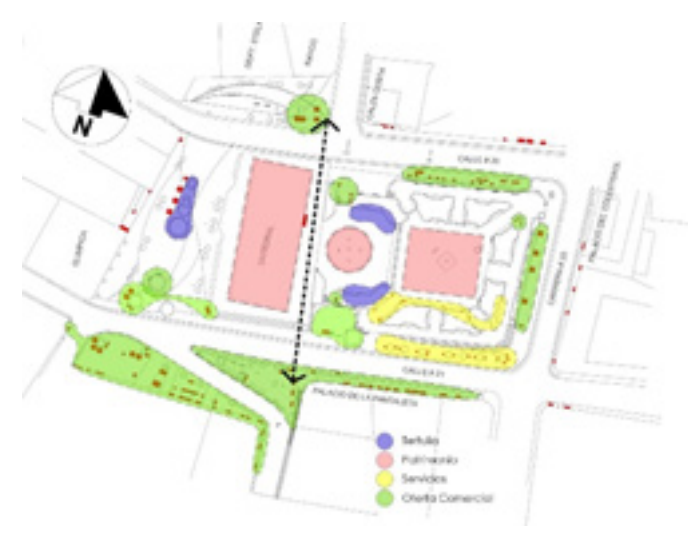

Figura 5: Plano de las dinámicas de flujo positivo en el centro histórico de Sincelejo

Fuente: Los autores (2016)

Algo de esto es posible verlo en las subcategorías que hacen parte de los "sentimientos sobre el lugar", en donde se referencia la sombra, frescura asociada con sentimientos de armonía, alegría, relajación y tranquilidad en el espacio público.

Por otra parte, en lo negativo, el ambiente es referenciado con relación al ruido, contaminación visual, problemas de aseo e higiene en el espacio público, e incluso la presencia de algunas especies animales dentro del espacio público.

Es interesante ver, desde esta relación, que el concepto "lo ambiental", en lo positivo está más enfocado hacia la buena percepción sobre los elementos naturales que hacen parte del conjunto de estructuras urbanas del centro histórico de Sincelejo y la vivencia de los mismos en el lugar. Por ejemplo, el arbolado urbano, el confort climático que este ofrece, el aire, la tranquilidad y la conservación de la naturaleza en el entorno urbano.
Dentro del proceso de análisis se distinguen tensiones entre las categorías y las percepciones que aparecen referenciadas por los habitantes en el espacio público del centro de Sincelejo. Lindón (2007) propone esas tensiones como un camino a la comprensión de imaginarios. En este caso las percepciones plantean desencuentros que llevan a entender los imaginarios que se construyen desde las representaciones de los habitantes.

Los imaginarios urbanos, como aquellas representaciones colectivas de los habitantes que apropian el lugar, se sintetizan de la siguiente forma: 1 . El centro histórico y el deseo de socialización; 2. El centro histórico como un espacio de insatisfacción del deseo y miedo.

Una de las prácticas urbanas que evidencia el imaginario sobre el centro histórico y el deseo de socialización, es el encuentro que se hace posible, sobre todo, en el parque Santander y la Plaza Olaya Herrera. De estos encuentros, es muy representativa la tertulia como actividad que hace posible la satisfacción del deseo de socialización en el lugar.

Por otra parte, las prácticas que evidencian el imaginario de insatisfacción y miedo en relación al centro histórico son, principalmente, el comercio informal llevado a cabo en las ventas estacionarias y ambulantes que ocupan el espacio público del centro, lo cual es visto de forma negativa por los habitantes. Asimismo, la presencia estratégica de prostitución, juegos de azar y consumo de licor en el espacio público, afectan negativamente la representación colectiva de la comunidad hacia el centro histórico; actividades urbanas estas que deterioran la imagen del lugar y contribuyen con la pérdida de identidad y la paulatina merma de la memoria de uno de los sectores de mayor importancia a nivel urbano de la ciudad.

Al observar el imaginario que habla sobre el centro histórico y el deseo de socialización, se identifica una presencia fuerte de la relación paisaje y habitante en la búsqueda del espacio público para socializar, ya sea en las bancas, alrededor de la fuente o en los arriates, donde se aprovecha la sombra de la vegetación para tener un lugar confortable en el que compartir, en el sentido del paisaje como la interrelación entre sociedad - cultura naturaleza.

Sobre esta relación habitante- paisaje, se pueden identificar aspectos interesantes de las motivaciones que la generan. Se podría destacar: el aprecio por la naturaleza, la búsqueda de descanso, reposo y calma, la frescura que ofrece la sombra de los árboles, y el encuentro de un ambiente propicio para conversar y escuchar amigos e historias de vida de las personas que se reúnen para compartir un rato.

Con relación al análisis de las condiciones ambientales y paisajísticas del centro histórico a partir del estudio de tipo cuantitativo desarrollado, se identifica, a través del registro de las sombras arrojadas por la vegetación y los objetos arquitectónicos en el área durante los primeros seis meses del año 2015, que efectivamente la vegetación influye en la conformación de microclimas en el centro histórico de Sincelejo. Específicamente, se puede hablar de las condiciones favorables que se 
observan en las zonas de arriates del Parque Santander, en donde se logra, por la presencia de vegetación, una disminución de la temperatura en un promedio de $6,6^{\circ} \mathrm{C}$ donde oscila entre $33,4^{\circ} \mathrm{C}$ temperatura promedio bajo sol y $26,8^{\circ} \mathrm{C}$ temperatura bajo sombra.

Es interesante ver cómo existen unas zonas con unas condiciones climáticas privilegiadas durante gran parte del año, producto de su ubicación estratégica y las condiciones que generan las especies arbóreas localizadas en los arriates del Parque Santander.

Es así que la zona de arriates ubicada al sur-oriente de la fuente del Parque Santander, goza de unas condiciones especiales, prácticamente con sombras durante gran parte del año. El Parque, con la totalidad de su arbolado, ofrece múltiples zonas confortables a diferentes horas del día, pero en la zona en mención se observa un uso e interés por el desarrollo de dinámicas urbanas especiales que en otras zonas con condiciones similares no es posible identificar con tanta fuerza.

Es interesante entender las dinámicas que suceden en el sector de arriates, al sur-oriente de la fuente en el Parque Santander, ya que es identificado como uno de los lugares en donde se desarrollan prácticas como la tertulia, que sustenta percepciones positivas y en consecuencia refuerza el imaginario del centro histórico y el deseo de socialización.

Esta relación evidencia una correspondencia en doble vía entre aspectos naturales y aspectos culturales de paisaje; ese lugar específico se convierte en un lugar de interacción constante en el cual la vegetación proporciona condiciones de bienestar térmico microclimático que son apropiadas mediante prácticas que construyen valores identitarios en la comunidad (figura 6).

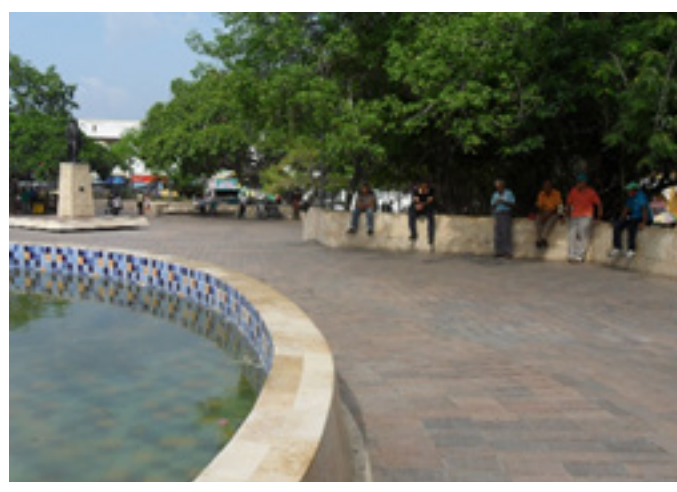

Figura 6: Fotografía de la tertulia en los arriates del parque Santander

Fuente: Los autores (2015)

\section{Discusión}

El trabajo realizado permite trazar líneas para la discusión, sobre todo en cuanto a entender la ciudad como organismo de carácter dinámico. En este caso se alcanza a evidenciar la interacción entre espacio público, paisaje y habitante, la cual permea las representaciones e imaginarios colectivos de la ciudad. Como una especie de integración entre el territorio y el habitante, uno y otro se impregnan del sentido que sus prácticas les inscriben. Pérgolis y Rodríguez (2013), plantean esta situación cuando explican que la ciudad, "con sus formas, usos y significaciones está en el interior" (p. 35). Se muestra ante el observador, pero la representación que se tiene y sus significados es lo que conforma el imaginario de ella.

En el mismo sentido, Beatriz García habla sobre el dinamismo de la ciudad. Afirma que es un hecho que se redefine y transforma permanente con las prácticas de sus actores (García, 2007; 2019). Para este caso, por las dinámicas del paisaje que lo condiciona, interactúa en diferentes caminos y construye sentido alrededor de lo humano y lo natural.

Esta especie de fusión habitante - ciudad, se da a través de la satisfacción del deseo de los que la utilizan, siguiendo las hipótesis trabajadas por Pérgolis (1998), cuando sugiere la ciudad como escenario capaz de satisfacer deseos de sus habitantes. Aquí, un deseo de interactuar con un tercero, acto motivado y a la vez aprehendido desde la relación entre el paisaje y el ser humano.

Las practicas urbanas, a través del tiempo y por la persistencia de su accionar en el lugar, se convierten en parte del paisaje mediante una integración en múltiples niveles con el espacio, el ser humano y la naturaleza, entendiéndose esas prácticas en el sentido complejo que describe Nogué (2010), cuando habla de "interrelaciones de carácter social y cultural, sobre una base natural, material" (p. 124).

Appleton (1996) habla sobre la teoría del paisaje como hábitat, afirmando que "la relación entre el observador humano y el medio ambiente que se percibe es básicamente la misma que la relación de una criatura a su hábitat" (p. 63). Para él esta relación es totalmente espontánea en cuanto que permite la oportunidad de lograr necesidades biológicas básicas, lo cual, al traerlo al contexto de este trabajo, puede plantear el interrogante sobre cuáles necesidades básicas satisfacen las dinámicas que se establecen en relación al paisaje y lo humano en el centro histórico de Sincelejo. Se puede afirmar que una de esas necesidades básicas es la satisfacción del deseo de integrarse en sociedad, aquello que distingue al ser humano como especie y hace capaces de amar y compartir en comunidad.

En ese sentido, el paisaje como elemento integrador o catalizador es de gran importancia para la consolidación de la identidad en un territorio (Nogué, 2010), de ahí que las interrelaciones que propicia puedan convertirse en patrimonio de una comunidad, en el sentido que propone Aponte (2008). En este caso particular, las dinámicas alrededor del paisaje pueden considerarse como un patrimonio cultural de la ciudad (UNESCO, 1972; 1977; 2003, 2005), una práctica que da sentido e identidad al lugar, en la cual se integran el ambiente, lo urbano y lo humano en la definición de uno de los espacios más representativos de la ciudad; evidencia empírica de lo que plantea Castells cuando explica que "el hombre se transforma y transforma su medio ambiente" (Castells, 2012, p. 141). Es decir, que tiene la capacidad 
de afectar su entorno, pero a la vez este le imprime un sello distintivo a su vida.

Las dinámicas que se desarrollan en el centro histórico de Sincelejo, en cuanto a la relación del paisaje y los imaginarios urbanos, como es el caso de las tertulias y el sentido de apropiación del espacio público y del lugar, pueden ser entendidas desde la noción de patrimonio cultural de la ciudad, ante lo cual se puede reflexionar sobre el legado como patrimonio que dejan estas dinámicas a las nuevas generaciones como fuente de identidad de una comunidad hacia el futuro.

Este trabajo da cuenta del sentido de apropiación de una parte de la ciudad, desde la relación entre el paisaje y las representaciones colectivas de sus habitantes, concepto que es trabajado también por Salazar (2010) desde una perspectiva cuantitativa con el fin de "crear instrumentos más rigurosos, capaces de superar una aproximación exclusivamente perceptual" (p. 13). Salazar, en el proceso se da cuenta de que la inclusión de aspectos sensibles en sus análisis ayudan a comprender mejor los comportamientos y actitudes de las personas con respecto a un lugar, e identifica que "no siempre la presencia de sombra se acompañaba de un fuerte uso por parte de las personas, contradiciendo las ideas previas acerca del tema" (2010, p. 22), interrogantes a los que da respuesta empírica este trabajo, ya que evidencia el sentido de lugar de las dinámicas en el espacio público.

En el sentido de la discusión que se proyecta, son muchos los interrogantes que se pueden plantear para ampliar la discusión en cuanto a las relaciones entre paisaje e imaginarios urbanos en busca de su valoración y preservación a futuro:

¿Cómo preservar prácticas urbanas que aportan identidad en la búsqueda de la resignificación de estructuras urbanas tan significativas como el centro histórico de Sincelejo?; ¿Qué particularidades de la relación entre paisaje e imaginarios se constituye en patrimonio de la ciudad con el fin de valorarlo y protegerlo?; ¿Cómo desarrollar políticas públicas de protección del patrimonio que incluyan los aspectos complejos de la relación entre paisaje e imaginarios urbanos en la ciudad?; ¿Cómo incorporar de forma activa a la universidad desde sus aspectos misionales y funciones sustantivos, en la valoración, salvaguarda y difusión del patrimonio cultural?

\section{Conclusiones}

A manera de cierre se plantean una serie de conclusiones a partir del ejercicio desarrollado.

Es innegable la relación entre la parte natural, espacial y física de la ciudad en cuanto a los servicios que ofrece al desarrollo de las dinámicas sociales en el lugar. Aquí es importante la relación entre el paisaje y los imaginarios urbanos, constituida por la correspondencia compleja entre diferentes componentes y las representaciones colectivas que dan sentido de lugar a uno de los espacios públicos más significativos en la ciudad.
El paisaje funciona como un catalizador para la satisfacción de los deseos de múltiples individuos. En este proceso, ser humano y paisaje se funden aportando sentido al lugar, lo que convierte a esta dinámica en un patrimonio a preservar y valorar en la ciudad.

Es muy importante la influencia de los paisajes urbanos en la consolidación de identidad y sentido del lugar en el contexto urbano, por ello es imprescindible tenerlos en cuenta al momento de realizar análisis para determinar elementos de valor patrimonial en la ciudad y el territorio. Más aún cuando en casos como el estudiado, las dinámicas que relacionan al paisaje y la memoria de sus habitantes se constituyen en un legado que, como paisaje cultural, se está dejando a las nuevas generaciones.

En las interacciones entre paisaje y habitante encontradas en el Parque Santander de Sincelejo, es posible ver cómo algunas prácticas y dinámicas urbanas, a través de su permanencia en el tiempo generan apropiación por parte de la comunidad, lo cual las lleva a convertirse en dinámicas representativas de los aspectos valiosos a destacar en la ciudad. Para algunos se convierten en prácticas que representan los imaginarios donde se satisfacen los deseos de muchos de sus habitantes; para otros, uno de los valores patrimoniales que como sociedad se tiene y que, debido a los constantes cambios en la estructura urbana, corren el riesgo desaparecer y quedar en el olvido.

\section{Referencias bibliográficas}

Aponte, G. (2008). El patrimonio natural como base del paisaje cultural en el entorno urbano. Libro de memorias del II Encuentro Internacional Restauradores Sin Fronteras A-RSF: Paisajes e itinerarios culturales como estrategias para el desarrollo. Bogotá, Colombia: Escala. Recuperado de http://www.sapcolombia.org/pdf/2013/ El\%20Patrimonio\%20Natural.pdf

Appleton, J. (1996). The landscape experience. Londres, Reino Unido: John Wiley and sons.

Benedict, M. A. y McMahon, E. T. (2012). Green infrastructure: linking landscapes and communities. Washington DC, USA: Islandpress.

Borie, M., Pelling, M., Ziervogel, G. y Hyams, K. (2019). Mapping narratives of urban resilience in the global south. Global Environmental Change, 54, 203-213. doi: 10.1016/j.gloenvcha.2019.01.001

Castells, M. (2012). La cuestión urbana. México DF, México: Siglo XXI editores.

Cidell, J. (2017). Sustainable imaginaries and the green roof on Chicago's City Hall. Geoforum, 86, 169-176. doi: 10.1016/j.geoforum.2017.09.016

De Certeau, M. (1996). La invención de lo cotidiano I. Artes de hacer. México DF, México: Universidad Iberoamericana. 
Gallardo Frías, L. (2012). De lo con-céntrico a lo descentrado. Reflexiones sobre el lugar y el no-lugar en la arquitectura. Revista de Urbanismo (26), 106 - 120. doi:10.5354/0717-5051.2012.20926

Gallardo Frías, L. (2013). Lugar y arquitectura. Reflexión de la esencia de la arquitectura a través de la noción de lugar. Arquitetura Revista, 9 (2) 161-169. doi:10.4013/ arq.2013.92.09

García-Canclini, N. (1997). Imaginarios urbanos. Buenos Aires, Argentina: Editorial Universitaria de Buenos Aires.

García Moreno, B. (2007). Ciudad, universidad y universitarios. Bogotá, el vecindario de la calle 45. Bogotá, Colombia: Editorial Pontificia Universidad Javeriana.

García Moreno, B. (2019). La ciudad de los habitantes y sus deseos. Procesos Urbanos, 6(6), 5-12. doi:10.21892/2422085X.451

Gorelik, A. (2002). Imaginarios urbanos e imaginación urbana: Para un recorrido por los lugares comunes de los estudios culturales urbanos. EURE, 28 (83), 125-136.

Gross, P., Buchanan, N. y Sané, S. (2019). Blue skies in the making: Air quality action plans and urban imaginaries in London, Hong Kong, and San Francisco. Energy Research \& Social Science 48, 85-95. doi: 10.1016/j. erss.2018.09.019

Jacobs, J. (2013). Muerte y vida de las grandes ciudades. Madrid, España: Capitán Swing Libros.

Lindón, A. (2007). La ciudad y la vida urbana a través de los imaginarios urbanos. EURE, XXXIII, (99), 7 - 16.

Maderuelo, J. (2005) El paisaje: génesis de un concepto. Madrid, España: Abada.

Martín- Barbero, J. (2002). Jóvenes: comunicación e identidad. Pensar Iberoamérica. Revista de cultura (0). Recuperado de: https://www.oei.es/historico/ pensariberoamerica/ric00a03.htm

Nogué, J. (2010). El retorno al paisaje. Enrahonar Quaderns de filosofía. (45), 123-136.

Pérez-Igualada, J. y Vicente-Almazán, G. (2020). Espacio, tiempo y paisaje. La representación de procesos y experiencias visuales en el análisis, el proyecto y la planificación de los espacios abiertos. Estoa. Revista de la Facultad de Arquitectura y Urbanismo de la Universidad de Cuenca, 9(17), 31-39. doi:10.18537/est.v009.n017. $\mathrm{aO} 3$

Pérgolis, J. C. (1998). Bogotá fragmentada. Cultura y espacio urbano a fines del siglo XX. Bogotá, Colombia: Tercer Mundo Editores.

Pérgolis, J. C. y Rodríguez Ibarra, C. I. (2013). E espíritu del tiempo en las ciudades y en sus libros. Revista de Arquitectura, 15 (1), 33-43. doi: 10.14718/ REVARQ.2013.15.1.4
Pérgolis, J. C. y Rodríguez Ibarra, C. I. (2014). El método en la investigación: imaginarios y representaciones de la forma urbana en la vida cotidiana. Procesos Urbanos, 1(1), 17-24. doi:10.21892/2422085X.14

Pérgolis, J. C. y Valenzuela, J. (2008). El método en dos investigaciones urbanas: Estación Plaza de Bolívar e Imaginarios y representaciones en el transporte público de pasajeros. Revista de Arquitectura, (10), 15-25.

Pérgolis-Valsecchi, J. C. (2020). Investigación en Historia: Ciudad, Memoria y Arquitectura. Procesos Urbanos, 7(1), 1-13. doi:10.21892/2422085X.484

Pérgolis Valsecchi, J.C. y Quijano Gómez, E. (2019). Memoria, acontecimiento, objeto y lugar. Procesos Urbanos, 6(6), 13-21. doi:10.21892/2422085X.453

P.O.T. (2015). Plan de ordenamiento Territorial de Sincelejo. Adoptado mediante decreto $N^{\circ} 438$ del 21 de agosto de 2015. Sincelejo, Colombia: Alcaldía Municipal de Sincelejo.

Rojo, F. y Enríquez, G. (2010). Imaginarios sociales urbanos vinculados a barrios cerrados en el Gran Concepción, Chile. Sociedad Hoy, (18), 65-83.

Salazar, J. H. (2010). Uso y apropiación de los espacios públicos. Medellín, Colombia: Universidad Nacional de Colombia.

Silva, A. (2006). Imaginarios urbanos. Bogotá, Colombia: Arango Editores.

UNESCO (1972). Convention Concerning the Protection of the World Cultural and Natural Heritage. Paris, France: UNESCO. Recuperado de: http://whc.unesco.org/ archive/convention-es.pdf

UNESCO (1977). 1st session of the World Heritage Committee. Paris, France: UNESCO. Recuperado de: http://whc.unesco.org/en/sessions/01COM/

UNESCO (2003). 27th session of the World Heritage Committee. Paris, France: UNESCO. Recuperado de: http://whc.unesco.org/en/sessions/27COM

UNESCO (2005). 29th session of the World Heritage Committee. Durban, South Africa: UNESCO. Recuperado de: http://whc.unesco.org/en/sessions/29COM 